\title{
Competitive Grain Growth and Texture Evolution during Directional Solidification of Superalloys
}

\author{
M.G.Ardakani, N.D'Souza, A.Wagner, B.A.Shollock and M.McLean \\ Department of Materials \\ Imperial College of Science, Technology and Medicine \\ Prince Consort Road, London, SW7 2BP, UK
}

\begin{abstract}
$\underline{\text { Abstract }}$
The development of crystallographic texture during directional solidification has been analysed quantitatively in columnar castings of the Ni-base superalloys, CMSX4 and CM186LC, produced with a range of cooling rates. The area density of grains having axial orientations within a specified scatter from $<001\rangle$ and the inverse pole figures (IPF's) were used to quantify the evolution of the axial texture. Increasing the solidification rate led to more effective competitive grain growth and a sharper $<001>$ texture for a given alloy. However, there are significant differences in the solidification characteristics of alloys with minor compositional differences; sharper $<001>$ textures were obtained in CM186LC than in CMSX4. The effects of solidification rate and composition on dendrite morphology have been investigated through quenched directional solidification experiments; the implications of these observations on the grain selection process and texture development are discussed.
\end{abstract}

\section{Introduction}

Casting of superalloy turbine blades in the directionally solidified and single crystal (SX) forms is now a wellestablished industrial process. However, recent developments in both alloy chemistry and in the dimension and complexity of castings have led to a concern about quality control; there is a trend to a more diffuse crystallographic texture during directional solidification, a wider range of single crystal orientations and more frequent occurrence of solidification defects such as spurious grains and freckles in Second and Third Generation alloys than in the earlier materials. The more important factors are:

- Recent developments in single crystal superalloy compositions have largely been directed to increasing the temperature capability of turbine blades and this has been achieved by progressively increasing the concentration of refractory solid solution strengthening additions, such as tungsten and rhenium. The improved high-temperature mechanical properties have been accompanied by an increase in the extent of microsegregation and significant changes in the solidification characteristics of the alloys.

- The dimensions of single crystal castings have progressively increased as the technology has been extended from small turbine blades for aero-engines to large castings for industrial gas turbines and to clusters of aero-engine parts. The larger mass of metal in these parts lead to quite different temperature distributions during casting. This leads to lower values of temperature gradient and solidification rate and to the possibility of there being a curved solid-liquid interface.

This paper addresses the role of the solidification rate and alloy chemistry on the evolution of texture; the role of interface curvature has been considered in earlier publications $[1,2]$. The study has been carried out on two commercial nickel-base superalloys; CM186LC is a variant of the earlier single crystal alloy CMSX4 and is specifically designed for use in the directionally solidified form. It is also being considered for production of single crystal blades to ensure that significant creep strength is retained, even when spurious grains are formed. In the first part of this paper, the evolution of axial texture in CM186LC under different local solidification conditions is considered; the second part is concerned with the grain selection process as a function of the alloy composition for a fixed set of the solidification parameters.

\section{Experimental}

\subsection{Directional Solidification Experiments}

Directional solidification (DS) of pre-cast ingots of $12 \mathrm{~mm}$ diameter encompassing a range of cooling rates was carried out using a Bridgman crystal growth apparatus with RF heating using graphite susceptors in an atmosphere of flowing argon. An important aspect of this investigation was the quantitative analysis of the competitive grain growth mechanism at curved liquidus isotherms which result from a radial component of the thermal gradient, in addition to the vertical component directed downward through the solid ingot to a watercooled copper chill plate. The DS rig is shown schematically in Figure 1 and details of the solidification conditions are listed in Table 1. Directional solidification was achieved by translation of a liquid metal zone of $\sim$ $30 \mathrm{~mm}$ length along the length of the initial charge. Adjustment of the radiation baffles at the base of the hot zone, which also corresponds to the position of the solidliquid interface, increased the radial component of heat flux from the solidified ingot. 
In industrial casting practice the entire charge is melted separately in a ladle in a vacuum or inert atmosphere and then poured into a shaped mould that incorporates a water-cooled chill. In the apparatus used for the present directional and single crystal solidification experiments, melting and solidification occur in a single chamber (Figure 1). The graphite susceptor, which lies within the central portion of the coils, directly couples with a copper induction coil to provide the heating source. However, because of the very high thermal gradients existing where the ingot contacts the chill, no melting occurred within $15-20 \mathrm{~mm}$ of the chill. Consequently, the oricntations of the pre-existing grains in the unmelted ingot were carried forward into the casting.

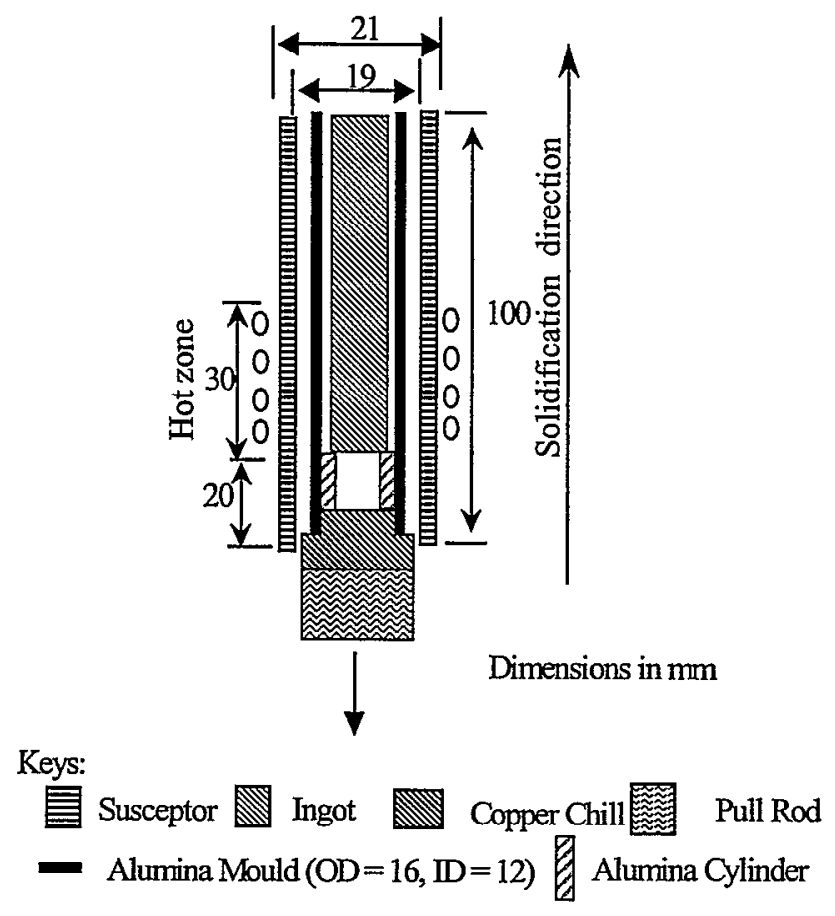

Figure 1. Schematic diagram showing configuration of solidification rigs used for directional solidification (Experiments 1-4, Table I).

In order to generate a truly random initial texture, similar to that occurring in industrial practice, the pre-cast ingot was supported on a hollow alumina cylinder of $12 \mathrm{~mm}$ diameter and $5 \mathrm{~mm}$ length to separate it from the chill. On melting the liquid metal flowed through the hollow gap and solidified on contact with the chill, thus nucleating a random axial crystallographic texture. The entire casting unit (mould with the charge and susceptor) was enclosed by a dense firebrick having a thermal conductivity of $0.1 \mathrm{Wm}^{-1} \mathrm{~K}^{-1}$. The initial charges in experiments 1 to 4 (Table I) had square cross sections of $7 \times 7 \mathrm{~mm}^{2}$. The initial length of the charge in experiment 1 was $200 \mathrm{~mm}$, while it was $130 \mathrm{~mm}$ for experiments 2,3 and 4.

The thermal gradient, $G_{L}$ at the solid-liquid interface and the solidification rate, $V$, are the most important parameters in determining the solidification structure. However, in practice, it is difficult to control these parameters independently in a DS apparatus. At steady state there is a balance between the heat supplied from the furnace, the latent heat generated on solidification and the heat lost to the chill. Considering only axial heat flow, this can be expressed:

$$
\mathrm{k}_{\mathrm{S}} \mathrm{G}_{\mathrm{S}}=\mathrm{k}_{\mathrm{L}} \mathrm{G}_{\mathrm{L}}+\Delta \mathrm{H}_{\mathrm{F}} \rho \mathrm{V}+\Delta \mathrm{EV}
$$

where $\mathrm{k}$ is the thermal conductivity, the suffixes $S, L$ represent solid and liquid respectively, $G_{L}$ is the thermal gradient at the solidification front, $G_{S}$ is the thermal gradient in the solid, $\Delta H_{F}$ is the latent heat per unit mass, $\Delta \mathrm{E}$ is the external energy provided to unit volume of the melt, $\rho$ is the density and $V$ is the solidification rate. $G_{L}$ and $V$ are clearly inter-related quantities which are not readily calculated, particularly when radial contributions to heat loss must be considered.

In the present DS set-up there are two important contributers to changing solidification conditions:

a. In addition to the heat conducted through the solid to the water cooled copper chill plate, the unmelted solid lying above the molten zone can radiate heat reducing the superheat in the melt. However, as solidification progresses and the length of the unmelted ingot decreases, this contribution to the melt heat loss is eliminated. This is results in an increasing melt temperature $\left(1580 \pm 20^{\circ} \mathrm{C}\right)$ in the final stage of DS, relative to that in the initial stages $\left(1470 \pm 20^{\circ} \mathrm{C}\right)$. This results in higher values of the temperature gradient in the liquid $G_{L}$ in the case of the shorter length ingots $\left(15-18 \mathrm{Kmm}^{-1}\right)$ than in the longer ingots $\left(11 \mathrm{Kmm}^{-1}\right)$.

b. As solidification progresses, the distance between the water-cooled copper chill and the liquidus front increases, thereby decreasing the axial temperature gradient in the solid $G_{S}$. In order to satisfy the energy conservation requirement, $V$ and $G_{L}$ must adjust to satisfy Equation 1.

The magnitude of the temperature gradient at the dendrite tips was estimated from measurements of the primary dendrite arm spacing (PDAS) $\lambda_{P}$ and the solidification rate $V$ by using the $G^{-0.25} V^{0.5}$ relationship that Qucsted and McLean [3] showed applied to a range of superalloys; this information is included in Table I. Solidification during single crystal production was interrupted by sudden quenching in a liquid metal bath in order to examine the development of the dendrite morphology in the mushy zone. Differential thermal analysis (DTA) was carried out on both alloys using a Stanton Redcroft STA-1780 Series simultaneous thermal analyser under an atmosphere of flowing argon. 
Table I: Directional solidification experiments.

\begin{tabular}{|c|c|c|c|c|c|c|}
\hline Exp. No. & Alloy & Nature & $\begin{array}{c}\text { Vx 10 } \\
\left(\mathbf{m s}^{-\mathbf{1}}\right)\end{array}$ & $\begin{array}{c}\text { Sol. Length } \\
(\mathbf{m m})\end{array}$ & $\begin{array}{c}\text { Primary dendrite } \\
\text { spacing }(\boldsymbol{\mu m})\end{array}$ & $\begin{array}{c}\mathbf{G} \\
\left({ }^{\mathbf{K}} \mathbf{K m m}^{-1}\right)\end{array}$ \\
\hline $\mathbf{1}$ & CM186LC & DS & 2.8 & 55 & $236 \pm 7$ & 5 \\
$\mathbf{2}$ & CM186LC & DS & 6.4 & 55 & $160 \pm 5$ & 7 \\
$\mathbf{3}$ & CMSX4 & DS & 2.8 & 35 & $197 \pm 27$ & 7 \\
$\mathbf{4}$ & CM186LC & DS & 2.8 & 35 & $201 \pm 7$ & 7 \\
$\mathbf{5}$ & CMSX4 & DS & 1.9 & 40 & $185 \pm 5$ & 10 \\
$\mathbf{6}$ & CM186LC & DS & 1.9 & 40 & $182 \pm 7$ & 10 \\
\hline
\end{tabular}

Table II: Alloy Composition (wt. \%).

\begin{tabular}{|c|c|c|c|c|c|c|c|c|c|c|c|c|c|}
\hline Alloy & Cr & Co & Mo & $\mathbf{W}$ & Ta & Re & Al & Ti & Hf & Ni & $\mathbf{C}$ & $\mathbf{T}_{\mathbf{L}}{ }^{\circ} \mathbf{C}$ & $\mathbf{T}_{\mathrm{S}}{ }^{\circ} \mathbf{C}$ \\
\hline CMSX4 & 6.5 & 9 & 0.6 & 6 & 6.5 & 3 & 5.6 & 1.0 & 0.1 & Bal & 0 & 1387 & 1347 \\
CM186LC & 6.0 & 9 & 0.5 & 8 & 3 & 3 & 5.7 & 0.7 & 1.4 & Bal & 0.07 & 1380 & 1320 \\
\hline
\end{tabular}

\subsection{Determination of Crystallographic Orientations using Electron Back Scattered Diffraction (EBSD) Patterns}

Specimens were prepared for metallographic and grain orientation characterisation by mechanical and electropolishing to remove any surface deformed layers produced during preliminary cutting, grinding and polishing. These samples were examined by means of the Electron Back Scattered Diffraction (EBSD) technique in a JEOL 840 scanning electron microscope, equipped with SINTEF hardware and CHANNEL+ software for automatic grain indexing and mapping. The maximum angular resolution was within $\pm 1.5^{\circ}$ and the spatial resolution was $1 \mu \mathrm{m}$ parallel to the specimen tilt axis. All of the grain orientations were specified with respect to a co-ordinate system based on the ingot geometry; the $\mathrm{Z}$ axis was parallel to the macroscopic growth direction; the $\mathrm{X}, \mathrm{Y}$ axes were parallel to arbitrary, orthogonal transverse directions that were identified on the grain maps that were constructed to show the spatial location of grains on a given section. The compositions of the $\mathrm{Ni}$-based superalloys, CMSX4 and CM186LC, used in this study are listed in Table II.

\section{$3 \underline{\text { Results }}$}

\subsection{Evolution of the Axial Texture}

\subsubsection{Role of the Local Solidification Parameters}

Figure 2 shows grain maps and inverse pole figures (IPF's) of surface normals of the grains on transverse sections taken at various distances along the length of a small commercial DS turbine blade of CM186LC. There is clearly both a progressive increase in grain size and the development of a sharp $<001>$ texture. Examination of longitudinal sections shows the establishment of a columnar grain morphology. The IPF for a solidification length of $85 \mathrm{~mm}$ shows that all of the grains have axial orientations within $5^{\circ}$ of $<001>$. Even after $2 \mathrm{~mm}$ of solidification the grain selection process has produced a diffuse texture with $90 \%$ of the axial orientations being clustered within $20^{\circ}$ of $\left.<001\right\rangle$. The thermal history (solidification conditions) of this blade is not known. However, a progressive coarsening of the primary dendrite arm spacing along the length of the blade is indicative of a diminishing cooling rate. Primary dendrite arm spacing $\left(\lambda_{\mathrm{P}}\right)$ corresponding to solidification lengths of $2 \mathrm{~mm}$ and $50 \mathrm{~mm}$ were $90 \mu \mathrm{m}$ and $383 \mu \mathrm{m}$ respectively. These measurements lack statistical significance for the thin cross section at a height of $85 \mathrm{~mm}$ because of an insufficient number of dendrites.

The purpose of the laboratory experiments described below is to characterise the grain morphology and texture development in different solidification conditions where the solidification parameters can be estimated. Figures $3 a$ and $d$ show grain maps from transverse sections after $55 \mathrm{~mm}$ of solidification in Experiments 1 and 2. There is a significant difference in the cross-sectional areas of the columnar grains and this is accompanied by a sharper $<001>$ texture being established in Experiment 2 than in Experiment 1 (Figures $3 \mathrm{~b}$ and $\mathrm{e}$ ). Figures $3 \mathrm{c}$ and $\mathrm{f}$ indicate the parts of the cross-section that have surface normals within $10^{\circ}$ of $<001>(95$ and $38 \%$ for Experiments 1 and 2 respectively). The primary dendrite spacings, $\lambda_{p}$ have been measured in both cases and are included in Table 1; the temperature gradients estimated from a knowledge of $\lambda_{\mathrm{p}}$ and $V$ are also included in Table 1. 


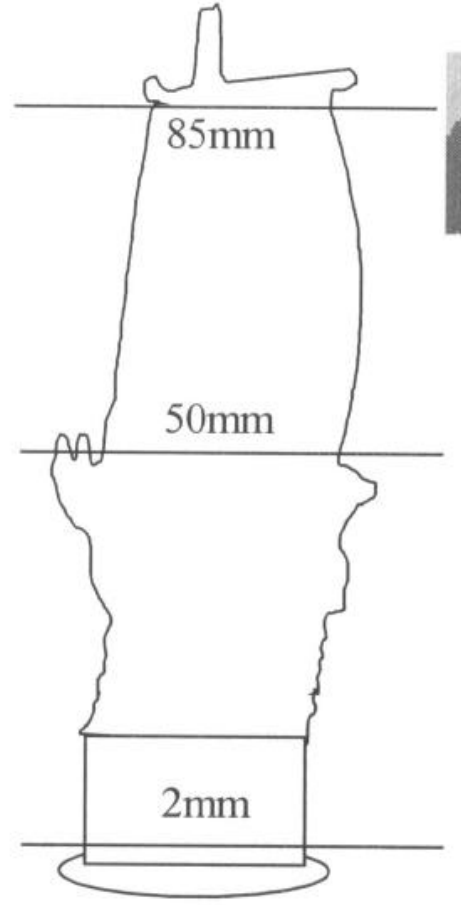

The Chill
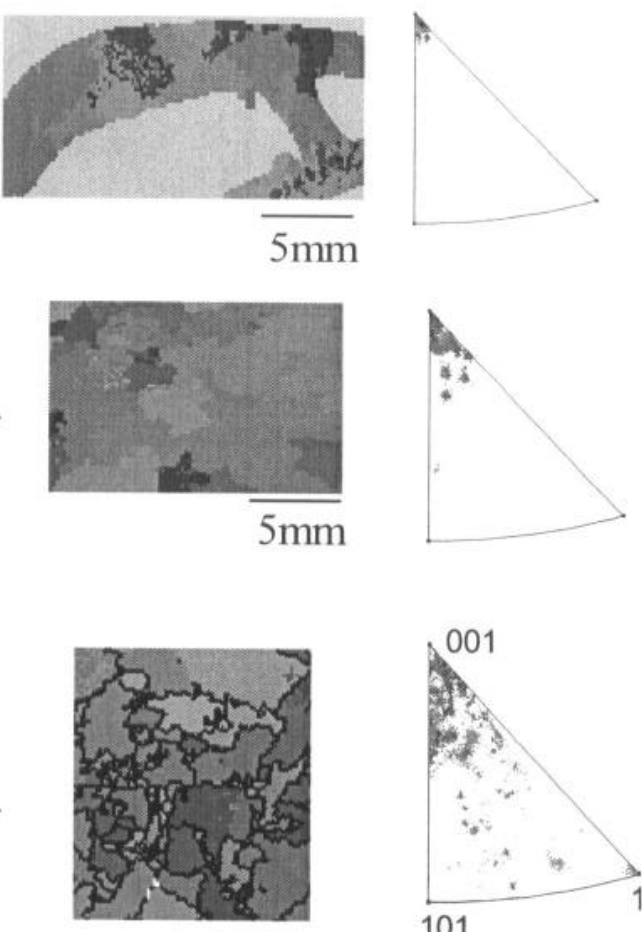

$3 \mathrm{~mm}$

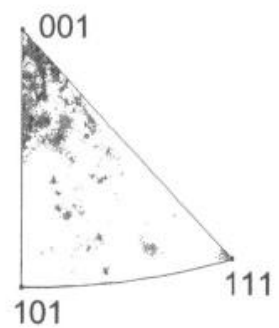

101

Figure 2. The transverse grain maps and inverse pole figures at different distances along a small directionally solidified turbine blade of CM186LC.

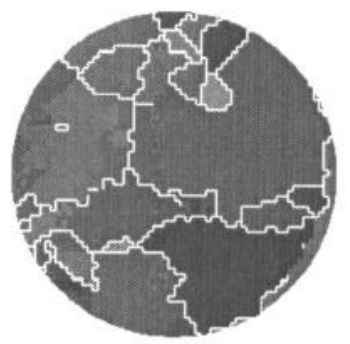

(a)

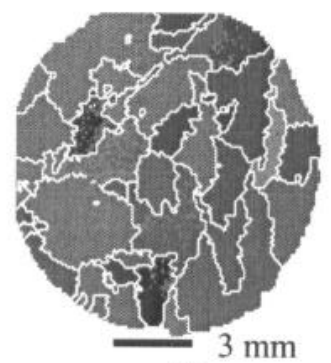

(d)

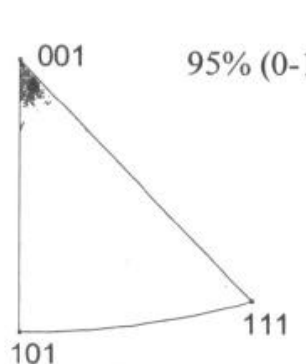

(b) 


\subsubsection{Role of the Alloy Chemistry}

The differences in composition of CM186LC and CMSX4 are quite minor (Table 2) and the physical constants that control heat flow and melting are also very similar. Consequently, it is expected that for the same ingot size and process conditions the principal solidification parameters will be identical. This expectation is supported by the observation of the same heating period before commencement of solidification for the two alloys; 18 minutes for Experiments 3 and 4. Consequently, any differences in grain or texture development is likely to be a consequence of the alloy constitution, rather than the solidification parameters, $G_{L}$ and $V$.

The authors have previously shown that under identical solidification conditions involving high temperature gradients, CM186LC develops a sharp $<001>$ texture whereas CMSX4 develops a diffuse texture (Figure 4 and Experiments 5 and 6 in Table 1). It is also noteworthy that for both alloys, the transverse grain diameters were 4 to $10 \mathrm{~mm}$, compared with 0.5 to $2 \mathrm{~mm}$ in Experiments 3 and 4 ; this indicates that grain selection was much more effective in Experiments 5 and 6. These earlier experiments were carried out without ensuring a totally random starting texture and consequently are not entirely comparable with Figure 4. Experiments 3 and 4, using the experimental procedures described above, were designed to investigate the grain selection process in solidification conditions that did not lead to a rapid establishment of a sharp $<001>$ texture in CM186LC.

Figure 5 shows grain maps, IPFs and the area of the crosssection with axial orientations lying within $7^{\circ}$ of $<001>$ for CMSX4 and CM186LC after a solidification length of $35 \mathrm{~mm}$ in Experiments 3 and 4. Unlike Figures $3 \mathrm{~b}$ and e, the IPF's do not show a clear difference in the axial textures of the two alloys. A more detailed comparison of the distribution of axial orientations for the two alloys was undertaken. The grain maps in Figures $5 \mathrm{c}$, f are sub-sets of Figures 5a, d respectively, which depict regions where the crystallites have axial orientations within a $7^{\circ}$ scatter from $\langle 001\rangle$. The area densities of grains are plotted as cumulative frequency as a function of angular deviation from $<001>$ in Figure $6 a$. The following deductions can be made:

1. A histogram plot of the area densities of grains as a function of axial misorientation shows a maximum for deviations of 10 to $15^{\circ}$ from $<001>$ (Figure $6 \mathrm{~b}$ ).

2. The plot of cumulative distribution of grain area as a function of misorientation shows that, even for the apparently diffuse textures obtained in Experiments 3 and 4 , the proportion of grains falling within $10^{\circ}$ of $<001>$ is significantly greater for CM186LC than for CMSX4. For CM186LC $10 \%$ and $90 \%$ of the crosssectional area is within $5^{\circ}$ and $20^{\circ}$ of $<001>$ respectively. For CMSX4, only $5 \%$ of the area has misorientations of less than $5^{\circ}$ and $30 \%$ of the area deviates from $<001>$ by more than $20^{\circ}$.
There is a clear implication from these observations that grain selection during directional solidification under identical experimental conditions, is more pronounced in CM186LC than in CMSX4.

The DTA results (Figure 7) show that the CMSX4 and CM186LC have similar thermal characteristics.

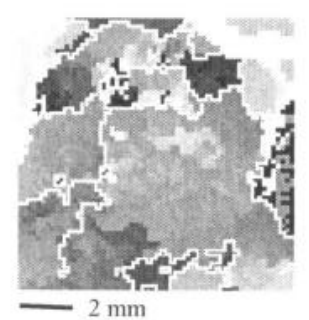

(a)

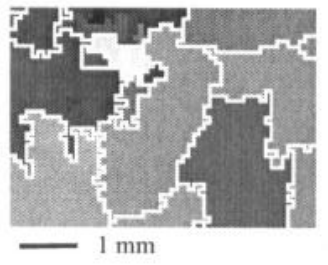

(b)
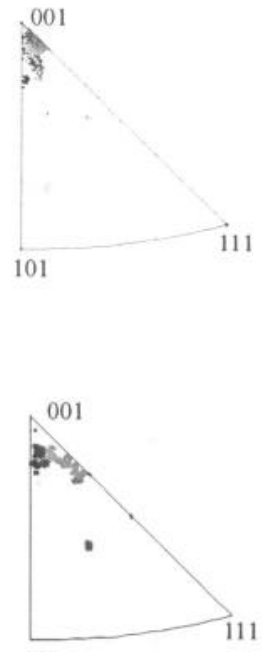

101
Figure 4. Grain maps and IPF's showing and axial orientation of grains on a transverse section after a solidification length of $40 \mathrm{~mm}$. (a) CM186LC (Experiment 6), (b) CMSX4 (Experiment 5).

CM186LC has a marginally lower liquidus temperature (by $7^{\circ} \mathrm{C}$ ), a significantly lower solidus temperature (by $27^{\circ} \mathrm{C}$ ) and a wider temperature range (by $20^{\circ} \mathrm{C}$ ) than CMSX4. The most significant difference in the shape of the DTA curves is the kink at $1350^{\circ} \mathrm{C}$ that is thought to be associated with the formation of carbides. Other thermal characteristics, such specific heat and thermal conductivity, are almost identical for the two alloys. Similar forms and weight of charge were used in the directional solidification experiments to ensure that the melting sequences were identical. For both materials melting was initiated after a period of 18 minutes (Experiments 3 and 4, Table I). Thus, any difference in the evolution of the axial texture can be directly attributed to the alloy solidification characteristics rather than to the process history.

Figure 8(c) shows a longitudinal section of CMSX4 in which single crystal growth in the $<001>$ direction has been interrupted by quenching in a bath of liquid metal. The ingot has been carefully aligned using the Laue Back reflection technique and then sectioned to ensure that the plane observed contains both the $<001\rangle$ growth direction and one of the $\langle 010\rangle$ transverse orientations. The field shown in Figure 8(c) shows the primary dendrite propagating ahead of the solid/liquid interface and secondary dendrites developing further back in the semisolid region. 


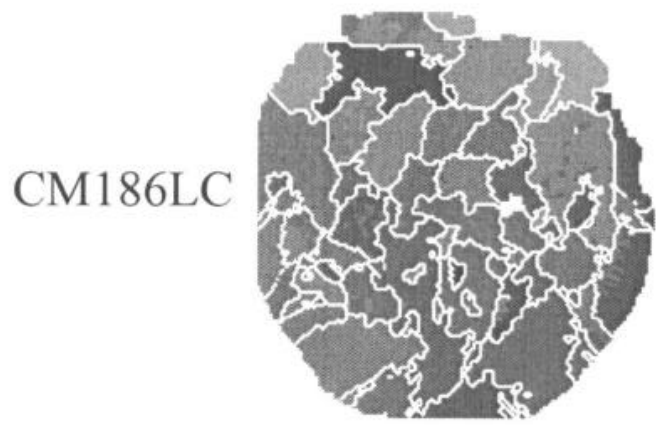

(a)

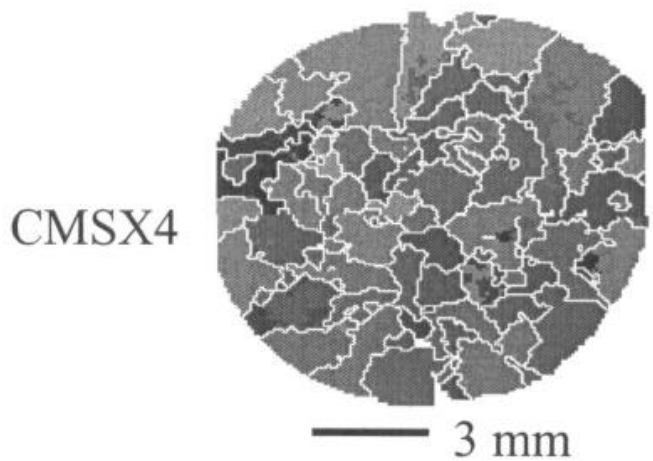

(d)

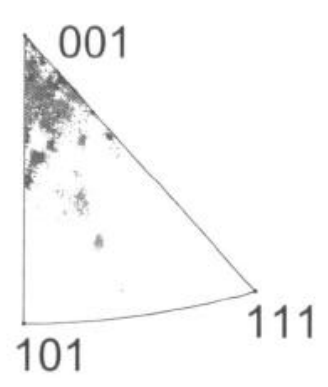

(b)

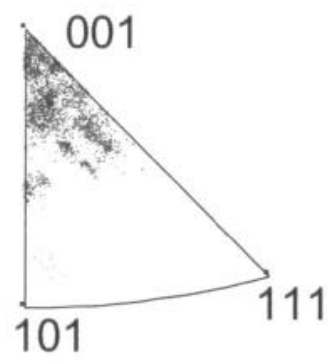

(e)

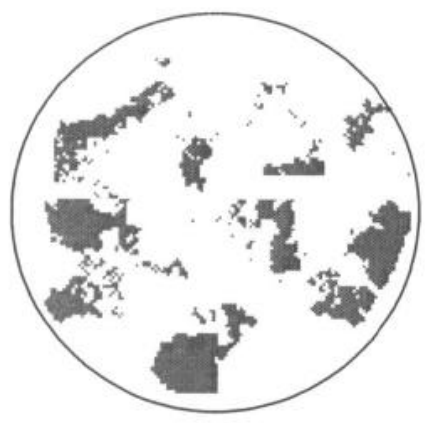

(c)

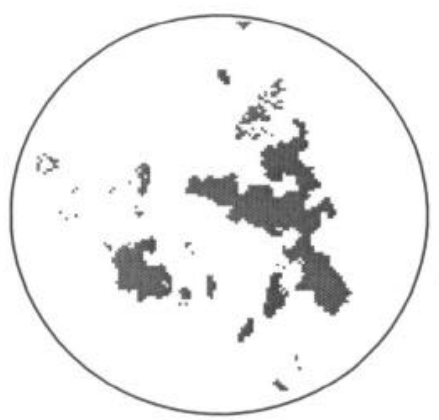

(f)

Figure 5. (a-c) grain map, IPF and distribution of grains showing spatial orientation of grains with respective axial orientations within $7^{\circ}$ from $<001>$, for CM186LC, after solidification length of $35 \mathrm{~mm}$, experiment 4 (Table I). (d-f) similar to (a-d) for CMSX4, experiment 3, (Table I)

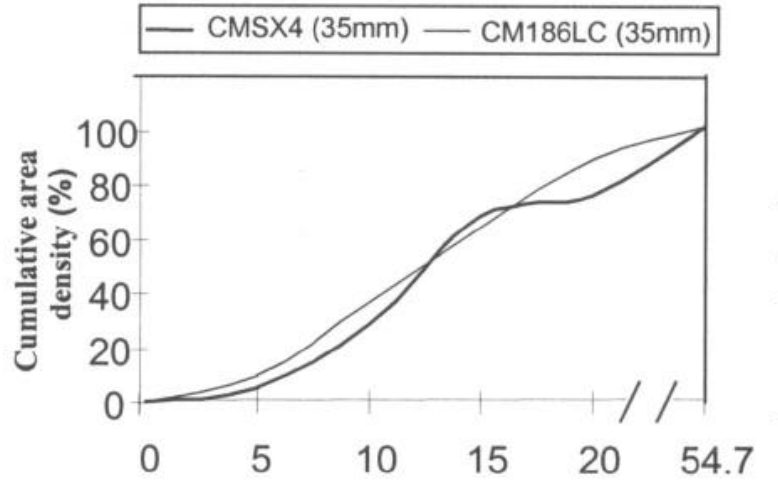

Deviation of axial orientations in Deg. from $<001>$

(a)

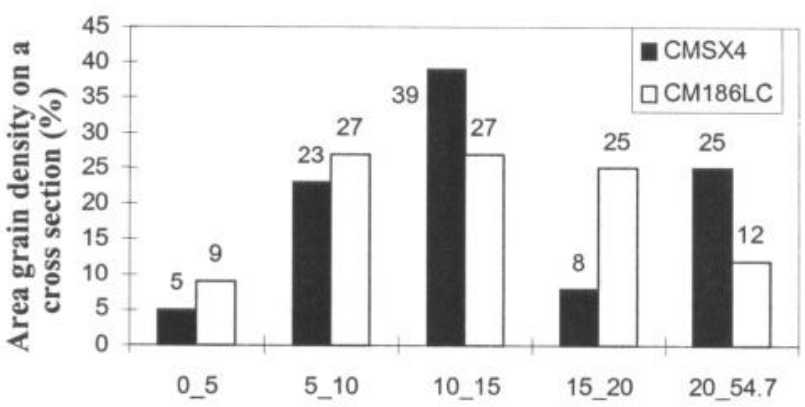

Deviation of axial orientations in Deg. from <001>

(b)

Figure 6. (a) Variation of the cumulative area density of grains with increasing angular deviation of the axial orientations from $<001>$, (b) Histogram plot showing the area density of grains in intervals of the angular deviation. 

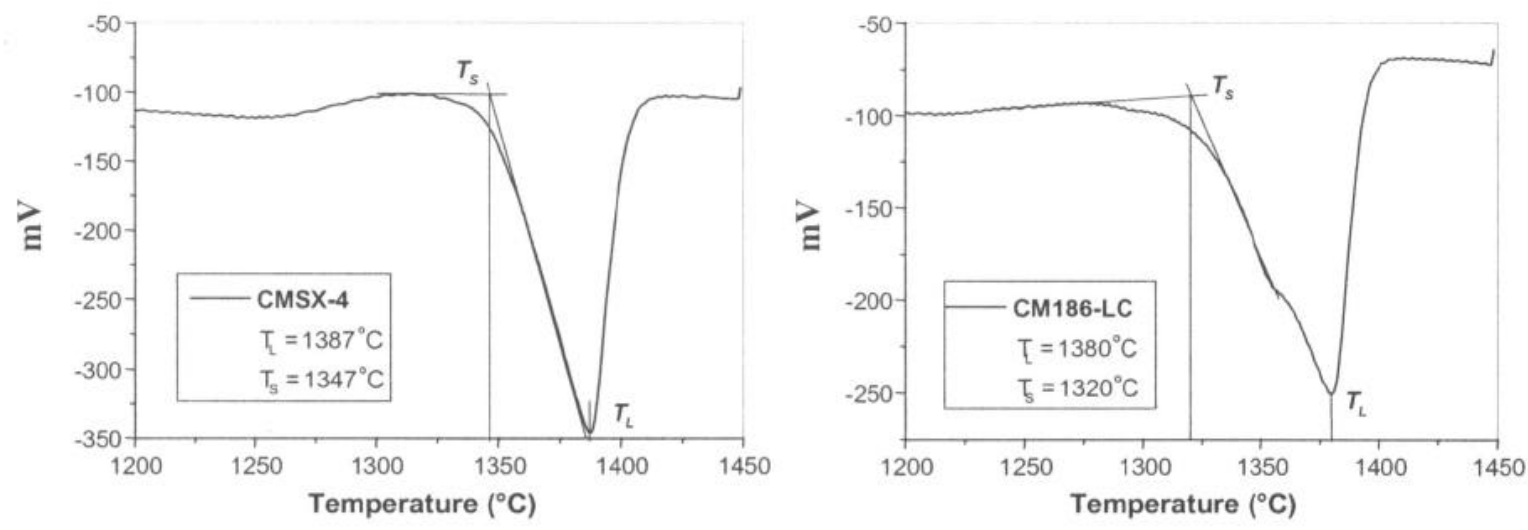

Figure 7. DTA curves for CM186LC and CMSX4 showing liquidus, solidus and carbide formation temperature.

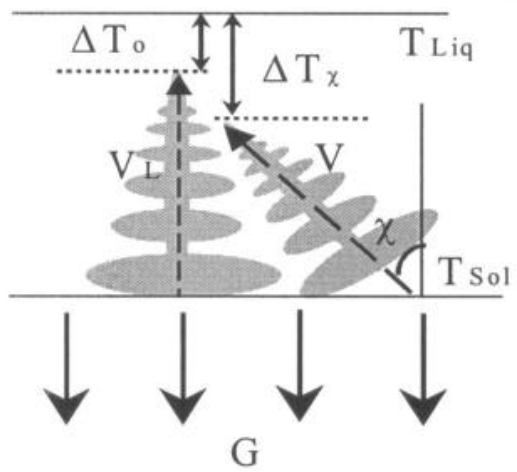

(a)

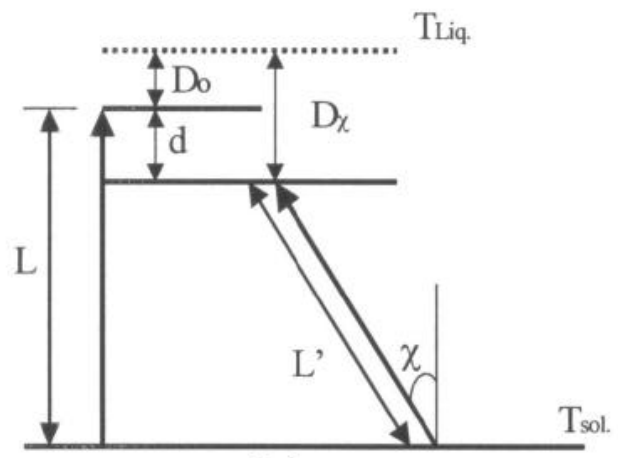

(b)

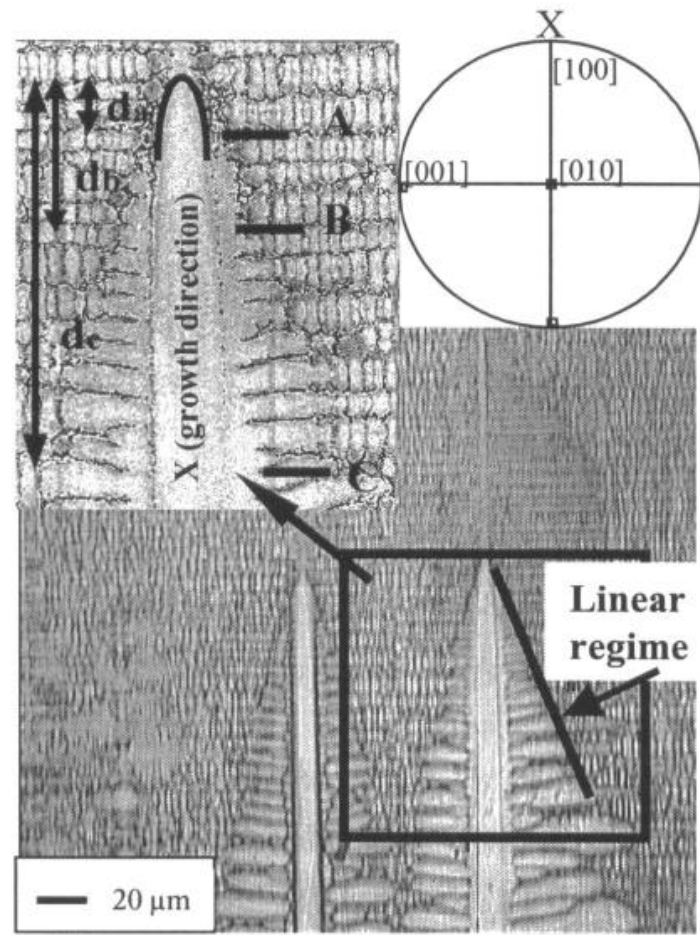

(c)

Figure 8. (a) Schematic diagram showing the tip undercoolings for favourably and unfavourably oriented dendrites with respect to the temperature gradient (G), (b) Estimation of the angle $\chi$ from geometrical and morphological constraints, (c) Optical and back scattered electron micrograph and (001) pole figure (inserted) showing the primary $<001>$ dendrite and the two regimes in the secondary arm morphology.

\section{Discussion}

\subsection{Nature of the Axial Texture}

It has been reported extensively that dendritic growth in cubic systems is parallel to $\langle 001\rangle$, although significant deviations have also been reported in some cubic metals (e.g. Cr) [6]. Nevertheless, the classical experimental observations of Walton et al .[7], involving the directional growth of $\mathrm{Al}$ and $\mathrm{Pb}$ alloys, show a clustering of the axial orientations close to $\langle 001\rangle$. They concluded that the evolution of a preferred crystallographic orientation, leading to an axial grain texture, was a consequence of the dendritic growth mechanism, and in particular was associated with the dendrite morphology, particularly that close to the dendrite tip. Specifically, it has been suggested that fast growing near $\langle 001\rangle$ grains block the development of slower growing mis-oriented grains by the growth of $<010>$ secondary dendrite from which $<001>$ 
tertiary dendrites nucleate and grow in the <001> macroscopic growth direction.

\subsection{Evolution of the Axial Texture}

\subsubsection{Role of the Local Solidification Parameters}

A theoretical treatment of the growth of an array of dendrites or cells in a positive thermal gradient has been given by Burden et al [8]. Neglecting interfacial attachment kinetics they express the net undercooling at the dendrite tips as:

$$
\Delta \mathrm{T}=\Delta \mathrm{T}_{\text {Sol }}+\Delta \mathrm{T}_{\text {Curv }}
$$

where the first and second terms represent the solutal and curvalure undercoolings respectively. The primary compositional contribution to the solutal undercooling is related to the diffusivity of the solute species in the melt ahead of the dendrite tip. Both alloys under consideration have similar concentrations of the highly segregating refractory solutes, rhenium and tungsten; they differ primarily in the levels of carbon and hafnium. It is likely that the solutal undercoolings will be dominated by the former, suggesting that there should be little difference between the alloys in this respect. The curvature undercooling, which has its origins in the Gibbs-Thomson parameter, depends on both the magnitude and anisotropy of the solid-liquid interfacial energy. Surface energies can be extremely sensitive to minor variations in alloy composition and these changes are not easy to predict. It is likely that this will be the causc of the significant differences in melting characteristics of the two, very similar alloys.

At steady-state, an unfavourably oriented dendrite with $<001>$ deviating from the direction of the local thermal gradient (G) by an angle $\chi$ can advance with the same vertical component of velocity $\left(V_{L}\right)$ as the liquidus isotherm, if it satisfies the condition:

$$
\mathrm{V}_{\mathrm{L}}=\mathrm{V} \cos \chi
$$

where $\mathrm{V}$ is the velocity of the primary dendrite parallel to its misoriented growth direction. Equation 3 can only be valid when $\mathrm{V}>\mathrm{V}_{\mathrm{L}}$. In the presence of a positive thermal gradient the unfavourably oriented primary dendrite lags the more favourably oriented one, and therefore grows with greater undercooling. It is the solute and thermal field of the leading primary that retards the growth of its lagging neighbour [7]. In all isothermal and nonisothermal dendrite growth theories, the tip supercooling $(\Delta \mathrm{T})$ and solidification rate $(\mathrm{V})$ can be expressed as [9]:

$$
\mathrm{V}=\mathrm{A}(\Delta \mathrm{T})^{\mathrm{n}}
$$

where $\mathrm{A}$ is a lumped material parameter that varies inversely with the solid-liquid interfacial energy and the exponent, $n$ lies in the range: $2 \leq \mathrm{n} \leq 3$.

For a given alloy, neglecting the temperature dependence of $A$, the difference in the undercooling between a primary dendrite tip aligned parallel to the local thermal gradient and another inclined at an angle $\chi$, as a function of the solidification rate, can be written as

$$
\Delta T_{\chi}-\Delta T_{0}=\sqrt[n]{\frac{V_{L}}{A}}\left(\frac{1}{(\cos \chi)^{1 / n}}-1\right)
$$

where $\Delta T_{\chi}$ and $\Delta T_{0}$ are the dendrite tip undercooling for the unfavourable and favourably oriented dendrite tips. These terms are shown schematically in Figures $8 \mathrm{a}$ in relation to the observed dendrite morphology. If $G_{L}$ is known this can be translated into a distance between the tips of well-oriented and mis-oriented dendrites:

$$
D_{\chi}-D_{0}=\frac{1}{G_{L}} \cdot \sqrt[n]{\frac{V_{L}}{A}}\left(\frac{1}{(\cos \chi)^{1 / n}}-1\right)
$$

It follows that for a given alloy ( $A$ constant), decreasing the growth rate $(V)$ leads to a smaller difference in the undercoolings at the tips of well- and mis-oriented dendrites. This in turn will make overgrowth of the misoriented dendrite by a secondary from the welloriented dendrite less likely and texture development more difficult.

Increasing the temperature gradient $\mathrm{G}_{\mathrm{L}}$ influences both the dimensions of the dendrites through the $G^{-0.25} V^{0.5}$ dependence of $\lambda_{P}$ and the morphology of the dendrite through the $G / V$ dependence of the dendrite/cellular/plane-front solidification transitions. The present results suggest that, providing that solidification is well within the dendritic growth regime, the primary dendrite spacing provides a good indicator of the effectiveness of grain selection; the finer dendrites lead to sharper solidification textures for a given alloy. However, this does not explain the differences between alloys.

In the solidification experiments of Huang et al [11] involving the transparent organic analogue succinonitrile, the formation of the initial secondary arms at the primary dendrite tips and their spacing (prior to coarsening in the mushy zone when the diffusion ficlds ovcrlap) is dependent on the dendrite tip undercooling. Therefore, instead of measuring the dendrite tip undercooling, the morphological aspects of the dendrite can be considered. This also assumes significance, since in the classical competitive grain growth process proposed by Walton $e t$ al [7], the growth of the tip of the unfavourably oriented dendrite, which lags the tip of the favourably oriented one, is retarded by the thermal and solutal environment of the latter. However, it is the secondary arms at the tip of the leading dendrite that eventually impair the further advancement of the lagging one beyond a certain length. Therefore, the morphology of these secondaries is crucial to the efficacy of the process of overgrowth. Therefore, from the perspective of competitive growth, the critical parameters would be:

a) The distance along the dendrite stem measured from the unbranched tip that the secondary arms form. Huang et al [9] observed an inverse relationship. 
b) The initial arm spacing $\left(\mathrm{S}_{\mathrm{Tip}}\right)$, before overlapping of diffusion fields leads to coarsening in the mushy zone.

c) The dendrite tip radius (R), which is a measure of the supercooling.

Since the dendrite blocking mechanism depends on effects in the vicinity of the dendrite tips, it is of interest to use the observed dendrite morphologies in Figure $8 \mathrm{c}$ to estimate the threshold value of $\chi_{\text {th }}$ at which mis-oriented dendrites are overgrown. $\mathrm{Li}$ and Beckermann [10] have shown from a detailed measurement in succinonitrile that the side-branch evolution is divided into two regimes: an initial linear regime and a subsequent non-linear coarsening regime. Our preliminary measurements support that interpretation; Figure $8 \mathrm{c}$ shows that the projection of the newly formed secondary arms (line A) to line $\mathrm{C}$ does in fact lie on a straight line. However, beyond line $B$, some non-linear coarsening is evident. In the following analysis we restrict consideration to this linear regime.

Overgrowth of a misoriented dendrite will occur if the distance between its and the well aligned dendrite tips $\left(D_{x}-D_{0}\right)$ exceed the distance $d$ along the dendrite at which significant secondary dendrites develop. Consequently a threshold value of $\chi$ can be identified below which no overgrowth will occur. Referring to the schematic diagram in Figure 8b:

$$
\cos \chi_{t h}=\frac{L-d}{L^{\prime}} \cong 1-\frac{d}{L}
$$

where $L$ and $L^{\prime}$ are the lengths of the two dendrites in the mushy zone (between $T_{L i q}$ and $T_{S o l}$ ) and $d$ is measured from the dendrite tip. For $d \ll<\mathrm{L}, \mathrm{L} \approx \mathrm{L}^{\prime}$ and equals the mushy zone length There is some arbitrariness in determining $d$, but taking distances along the dendrite to positions A. B and C shown in Figure $6 \mathrm{c}$ leads to estimates of $\chi_{\mathrm{th}}$ :

$$
\begin{aligned}
& d_{a}=35 \mu \mathrm{m}, \chi_{A}=5^{\circ} \\
& d_{b}=55 \mu \mathrm{m}, \chi_{B}=6^{\circ} \\
& d_{c}=110 \mu \mathrm{m}, \chi_{C}=9^{\circ}
\end{aligned}
$$

There is now a solid body of evidence pointing to the solute and thermal fields of the secondaries of the leading dendrite retarding the growth of a lagging neighbour. The observed morphology in Figure 8c of the quenched dendrite in CMSX4, solidified with conditions ( $\mathrm{V}=5 \times 10^{-5}$ $\mathrm{ms}^{-1}, \mathrm{G}=4 \mathrm{Kmm}^{-1}, \lambda=225 \mu \mathrm{m}, \mathrm{L} \approx 10 \mathrm{~mm}$ ) is consistent with a final axial texture having a scatter of up to $10^{\circ}$ from $<001\rangle$, which is reasonably consistent with casting experience.

The histogram shown in Figure $6 \mathrm{~b}$ indicates that there is an increasing population of grains with increasing misorientation until $\chi$ is about $10^{\circ}$. This is quite consistent with the above analysis. If all grains with $\chi<10^{\circ}$ can grow without being overgrown and if they originate from random distribution of grains then there are more possible orientations deviating from than with a precise $<001>$ direction. For this regime of no selection the probability increases as $(1-\cos \chi)$ which is approximately linear for $\chi<10^{\circ}$. Grains with $\chi>10^{\circ}$ are predicted to be overgrown leading to a reduction in frequency.

A detailed comparison of dendrite morphologies in a range of directionally solidified and single crystal alloys will be presented in a future publication.

\subsubsection{Role of the Alloy Chemistry}

The results presented above show that CM186LC and CMSX4 have a significant difference in axial texture resulting from identical processing conditions. The similarity in physical constants will lead to the same local solidification conditions $\left(G_{I,}, V\right)$. It follows that the observed difference in the axial textures can only be attributed to differences in thermodynamics and kinetics of dendrite growth, that is represented by the parameter, $A$. In the simplified model of Burden and Hunt. [8], the lumped material parameter, $A$ varies inversely as $\frac{\gamma_{\mathrm{SL}}}{\mathrm{D}}$, where $D$ is the solute diffusivity in the melt at the solidification front. For a multi-component system it is reasonable to take the diffusion coefficient of the slowest diffusing species, which in this case would be Ta, W and $\mathrm{Re}$. The role of surface energy is more difficult to assess since both the absolute value and the anisotropy are very sensitive to minor changes in chemistry. CM186LC and CMSX4 have similar concentrations of refractory solutes, which segregate to the dendrites, suggesting that the solutal terms will be similar.

There has been a common view based on experimental evidence that the newer generation of SX superalloys (CMSX4, CMSX10) are less tractable to orientation control than some new alloys that have been specifically developed for use in the DS form (CM186LC). The major difference in the alloy composition, is the presence of $\mathrm{Hf}$ and $\mathrm{C}$, both being added as grain boundary strengthening agents (Table $\mathrm{I}$ ). However, $\mathrm{C}$ forms carbides and the liquid at the solidification front in CM186LC will be enriched in $\mathrm{C}$ only if the carbides are formed below the liquidus front. This is indeed the case from the DTA plot in Figure 4. The carbides form between 1350 and $1360^{\circ} \mathrm{C}$, which for an average thermal gradient of $10 \mathrm{Kmm}^{-1}$, is 2$3 \mathrm{~mm}$ behind the dendrite tip. Therefore, the melt into which the dendrite tips are advancing is enriched in C. Experimental determination of $\gamma_{\mathrm{SL}}$ has usually been undertaken on transparent systems [11, 12] and such measurements at the high liquidus temperatures and in opaque systems are formidable and fraught with inaccuracy. 


\section{Conclusions}

1. The effectiveness of grain selection during directional solidification of a superalloy is sensitive to the processing conditions. High temperature gradients and solidification rates lead to a sharper $<001>$ texture and larger transverse grain diameters than low cooling rates. There is an inverse correlation between primary dendrite size and grain selection efficiency.

2. CM186LC develops a sharper $<001>$ axial texture than docs CMSX4 when processed under identical conditions. Even in conditions when both alloys produce diffuse textures, detailed analysis of area densities of grains with specific orientations shows that grain selection is more effective in CM186LC.

3. Analysis of the evolving morphology of the dendrite structure at a quenched solid-liquid interface indicates that misorientation of up to $10^{\circ}$ will not be overgrown by secondary dendrites. This is consistent with the observation of a peak in orientations at about $10^{\circ}$ misorientation.

4. DTA analysis shows that the carbides form $2-3 \mathrm{~mm}$ behind the dendrite tips in CM186LC showing that the carbides have no significant role in the grain selection mechanism. There is a possible role of $\mathrm{C}$ being rejected into the melt at the dendrite tips modifying $\gamma_{\mathrm{SL}}$ -

\section{Acknowledgements}

The authors thank ALSTOM ENERGY (formerly European Gas Turbines) for provision of a studentship (ND'S) and EPSRC for support (Grant No.GR/L05433).

\section{List of References}

1. N. D'Souza, M. G. Ardakani, B. A. Shollock, M. McLean, International Synposium on Liquid Metal Processing and Casting, eds. $\Lambda$. Mitchell, L. Ridgway, M. Baldwin, American Vacuum Society, Santa Fe, 1999, p.1.

2. N. D'Souza, M. G. Ardakani, B. A. Shollock, M. McLean, Met. Trans. A, in press.

3. P.N. Quested and M. McLean: Mat. Sci. Eng. 1984, vol. 65, p. 171.

4. D. A. Porter, K. E. Easterling, Phase Transformations in Metals and Alloys, $2^{\text {nd }}$ edition, 1992, Chapman and Hall, p. 204.

5. F. Giamei, J. G. Tschinkel, Met. Trans, Vol 7A,1976, p. 1427.

6. D. N. Lee, K-H Kim, Y-G Lee, C-H Choi, Materials Chemistry and Physics, 1997, vol. 47, p. 154.

7. D. Walton and B. Chalmers: Trans. Met. Soc. of AIME. 1959, vol. 215, p. 447.

8. M. H. Burden and J.D. Hunt: J. Crystal Growth. 1974, vol. 22, p. 109.

9. M. E. Glicksman, R. J. Schaefer, J. D. Ayers, Met Trans. A, vol 7A, p. 1747.

10. Q. Li, C. Beckermann, Acta Mater., 1999, vol. 47, p. 2345.

11. S-C. Huang and M.E. Glicksman: Acta Met. 1981, vol. 29, p. 717.

12. D. R. H., Jones, G. A. Chadwick, Phil Mag., 1970, vol., p. 291. 